\title{
Immobilization of Gold and Silver on a Biocompatible Porous Silicone Matrix to Obtain Hybrid Nanostructures
}

\author{
Solano-Umaña Victor ${ }^{1}$, Corrales Urena Yendry Regina², Vega-Baudrit José Roberto² \\ ${ }^{1}$ Hologic Surgical Products Costa Rica, Alajuela, Costa Rica \\ ${ }^{2}$ National Laboratory of Nanotehcnology (LANOTEC-CeNAT-CONARE), San José, Costa Rica \\ Email: victor.solano@hologic.com
}

How to cite this paper: Victor, S.-U., Regina, C.U.Y. and Roberto, V.-B.J. (2018) Immobilization of Gold and Silver on a Biocompatible Porous Silicone Matrix to Obtain Hybrid Nanostructures. Journal of Biomaterials and Nanobiotechnology, 9, 41-50. https://doi.org/10.4236/jbnb.2018.91004

Received: October 17, 2017

Accepted: January 8, 2018

Published: January 11, 2018

Copyright $\odot 2018$ by authors and Scientific Research Publishing Inc. This work is licensed under the Creative Commons Attribution International License (CC BY 4.0).

http://creativecommons.org/licenses/by/4.0/

\begin{abstract}
During the last decade an enormous research effort has been deployed with respect to porous materials. Design, pore size, shape, morphology and density are crucial features for increasing the surface area of silicone materials, aiming for a better biological response so cells can adhere and grow. Many medical applications utilize polydimethylsiloxane (PDMS) in medical implants, despite its hydrophobic surface that does not stimulate cellular adhesion. Porosity and morphology are important factors in the wettability of PDMS, but modifying the hydrophobic surface functionalization is required. To achieve this goal, the use of coatings with gold and silver nanoparticles or nanofilms can be used as a strategy to improve biocompatibility. This is due to the effect on mammalian cell adhesion and proliferation related to gold nanoparticles, as well as the prevention of infections related to silver nanoparticles. In this study, the pores in the silicone matrix were formed using sugar crystals as a template agent, and later passed through a lixiviation process to form a porous silicon matrix. Next, the matrix was placed inside a colloidal suspension; a process that allowed the immobilization of these particles on the surface matrix. A hybrid stable material was synthetized through this process. The water absorption level of the porous silicone matrix with and without the nanoparticles was determined. The water uptake of the matrix was higher when the nanoparticles were immobilized on the surface. Van der Waals and hydrogen bonding interactions account for this, promoting the retention of a higher concentration of water molecules. Higher water uptake has been identified as being a key factor for improving biological response, cellular adhesion and growth, which accelerates implant integration in the body.
\end{abstract}

\section{Keywords}

Silicone, Porous, Gold, Silver, Water Absorption 


\section{Introduction}

Silicone based materials have been used in medical devices and implants because of their biocompatibility and low cost [1]. Medical devices such as catheters and breast implants are examples of uses in the medical industry [2] [3]. Therefore, materials containing silicone have been relied on to play an important role in bone health, due to collagen synthesis promotion and possible matrix mineralization [1].

One disadvantage of silicone matrices is limited in growth. The hydrophobicity of the silicone matrix surface could negatively influence implant acceptance. Approaches based on surface modifications with peptides, proteins and different functional groups and/or nanoparticles have been used for changing the behavior of the surface in terms of surface reactivity, rigidity and characteristics such as quantum confinement effects [4]. Nowadays, many studies are dealing with understanding the interaction between the material and mammalian cells in a physiological environment. Improving biological response is a complex process that still requires a lot of research [5].

Silver and/or gold have been deposited on silicone surfaces using different techniques such as immersion-plating [6], drop casting [7], and dropping a colloidal solution on the surface [8] with sputtering [9]. In this study gold and silver nanoparticles were synthesized in situ and immobilized on a porous silicone matrix to improve biocompatibility and antimicrobial properties. In general, metal nanoparticles on silicone surfaces have been proposed to improve vascularization and cellular growth over and inside the implant [10].

Gold nanoparticles have been used as a material which improves implant biocompatibility. The strong binding of peptides and antibodies improves cell adhesion [10]. On the other hand, silver nanoparticles provide antimicrobial properties [11]. The synergy of nanoparticles immobilized on a surface has been chosen as a strategy to diminish the body's rejection due to low wettability and risk of bacteria colonization [12]. The control of the surface properties at the interface plays the main role; chemistry, topography and wettability determine a good or bad biological response [13]. It has been theorized that tissue repair after implants is dependent on a wet environment [14]. The present investigation focused on water absorption behavior changes of a porous silicone matrix due to nanoparticle immobilization. Porous silicone matrix and nanoparticle synthesis has been described in more detail by the authors in previous publications [5] [15].

Techniques such as scanning electron microscopy (SEM), optical microscopy and water absorption tests were used to characterize the material.

\section{Experimental}

The experimental methods Section 2.1 follows the experimental steps described in a previous paper for which the goal was to maintain the original porous silicone matrix pore size from 100 to 300 micrometers. This goal was addressed using gold and silver nanoparticles size control though chemical reaction parameters: concentration, $\mathrm{pH}$ and temperature. Metal particle nanometer size does not affect the porous silicon matrix pore size [16]. 


\subsection{Preparation}

\subsubsection{Chemicals}

Porous silicone matrix was prepared in the laboratory with poly-dimethylsiloxane (PDMS) product of Nusil Silicone technology (MED-4860) the pore size are between 0 and 300 micro meters, $\mathrm{AgNO}_{3}$ (99.99\%, Sigma-Aldrich), $\mathrm{HAuCl}_{4} \cdot 3 \mathrm{H}_{2} \mathrm{O}$ (99.99\%, Sigma-Aldrich), L-Ascorbic acid (99\%, Sigma-Aldrich), disodium ethylenediaminetetra-acetate (EDTA-2Na ) ACS reagent (99.4\%, powder, Sigma-Aldrich), dextrose monohydrate (USP grade, Sigma-Aldrich), sodium hydroxide (ACS reagent, $\geq 97.0 \%$, pellets, Sigma-Aldrich), ammonium hydroxide solution (ACS reagent, $28.0 \%-30.0 \%$, Sigma-Aldrich), and anhydrous sodium Sulfite ( $\geq 98 \%$, Sigma-Aldrich).

To prepare a chemical solution and perform silver and gold immobilization treatment over the porous silicon matrix see previous papers, and diagram, Figure 4.

\subsubsection{Porous Silicone Matrix}

The porous silicone matrix was prepared by molding and injection. The sugar was sieved to control the crystal size from 100 to $300 \mu \mathrm{m}$. A $5 \% \mathrm{~m} / \mathrm{m}$ (DI-water/sieved sugar) was prepared. The mold was filled up with this mixture. A small channel was opened in the middle of the sugar mold. Then, the silicone was mixed with heptane in a proportion 80/20 and this mix was injected into the internal channel of the sugar mold. The silicone was cured at $80^{\circ} \mathrm{C}$; the mold was placed in deionized water at $50^{\circ} \mathrm{C}$ and stirred to remove the sugar from the silicone matrix.

\subsubsection{Silver Solution}

The $\mathrm{AgNO}_{3}$ was dissolved in DI-water and ammonium hydroxide was added (concentrated ammonia, specific gravity 0.88 ) to form an ammoniacal silver complex $\left(\left[\mathrm{Ag}\left(\mathrm{NH}_{3}\right)_{2}\right] \mathrm{OH}\right)$ final concentration $0.0135 \mathrm{M}$. Next, the EDTA-2Na solution was added, molar ratio 1:1 respect to $\mathrm{AgNO}_{3}$. The porous silicone matrices were added to a silver solution. Next, a dextrose solution was added and heated to reach a temperature of $50^{\circ} \mathrm{C}$ for one hour. The molar ratio from silver nitrate to dextrose monohydrate was $1: 5$, final $\mathrm{pH}=9.0$.

\subsubsection{Gold Solution}

The $\mathrm{HAuCl}_{4} \cdot 3 \mathrm{H}_{2} \mathrm{O}$ was dissolved in DI-water and sodium sulfite $\left(\mathrm{Na}_{2} \mathrm{SO}_{3}\right)$ was added in a molar ratio of 1:10. Next, the EDTA-2Na solution was added, molar ratio 1:1 respect to $\mathrm{HAuCl}_{4} \cdot 3 \mathrm{H}_{2} \mathrm{O}$. The porous silver/silicone matrices were mixed with $\mathrm{HAuCl}_{4} \cdot 3 \mathrm{H}_{2} \mathrm{O}$ solution. Next, the ascorbic acid was added in a molar ratio of 1:10 with respect to $\mathrm{HAuCl}_{4} \cdot 3 \mathrm{H}_{2} \mathrm{O}$. Finally, the solution was heated at a temperature of $50^{\circ} \mathrm{C}$ for one hour, final $\mathrm{pH}=9.0$.

\subsection{Characterization Method}

\subsubsection{Optical Inspection}

A visual inspection was performed using a Smart Scope Flash 200, model CNC200, serial SVW2003849. Smart Scope Flash is an automatic dimensional piece of 
equipment, with a measurement system, and optical metrology that uses a large capacity video system for verification and control of dimensional manufacturing parts.

\subsubsection{Fluorescence $X-$ Ray Spectrophotometer}

Silver and gold immobilization over the surface was detected using an X-ray fluorescence (XRF) spectrometer. The Fischerscope XRF Model XDLM-C4 is used for routine analysis of different materials. Additionally XRF is a non-destructive method and this analytical technique can also determine the elemental composition of materials. This analytical equipment determines the chemistry of a sample by measuring the fluorescence emitted from the material surface when it is excited by a primary X-ray source. Each metal from the sample produces characteristic fluorescent X-ray patterns, or fingerprints, unique for that specific metal. Therefore the equipment is capable of giving qualitative and quantitative analysis from the sample.

\subsubsection{Scanning Electron Microscopy (SEM)}

SEM images were obtained, using a JEOL JSM-6390LV Scanning Electron Microscope, pore and particle size were verified.

\subsubsection{Water Absorption}

An analytical scale was used to measure the deionized water absorption percentage of the porous silicone matrix. The dried matrix samples were weighed, placed in deionized water, and mixed for $2 \mathrm{~h}$. Next, the samples were removed and dried at environmental conditions. Later the porous silicone matrix was weighed again to establish the percentage of water absorbed. The pure porous silicone matrix was compared with a different porous silicone matrix that has $\mathrm{Ag} / \mathrm{Au}, \mathrm{Ag}$ and $\mathrm{Au}$ nanoparticles immobilized on its surface.

\subsubsection{Wettability Test}

A contact angle goniometer was used to measure the wettability of silicone film with and without $\mathrm{Ag} / \mathrm{Au}$ immobilized nanoparticles. The silicone film was mixed with the silver solution as described in Sections 2.1.3 and 2.1.4. Then the contact angle was measured on the pure silicone film and $\mathrm{Ag} / \mathrm{Au}$ silicone film.

\section{Results}

The sugar or pore forming agent was sieved to select the crystals with a size below $300 \mu \mathrm{m}$. Figure 1(A) and Figure 1(B), shows the images taken by a Smart Scope of the sugar crystals after sieving and separating the large crystals. All crystals used are equal or below $300 \mu \mathrm{m}$, Figure 1(A).

Figure 2 shows the pore size distribution with a mean pore size of $141 \mu \mathrm{m}$. The pore-forming agents play an important role. They are crystals that could be removed with a solvent such as water, and crystal size will influence the pore size obtained. There is a direct relation between pore-forming crystal size and the pore size obtained in the matrix [15].

Pore size, connectivity and density were observed on the scanning electronic 


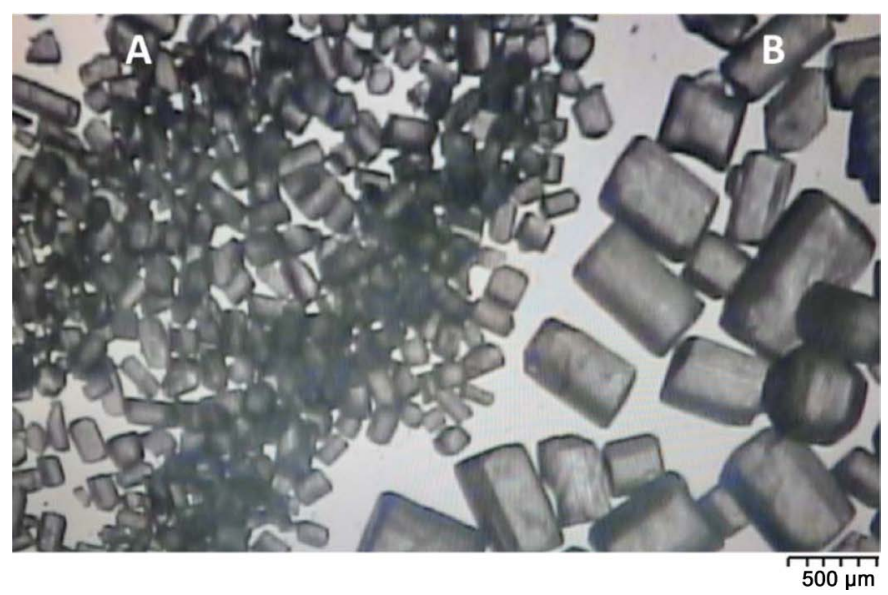

Figure 1. Forming pore agent (sugar) after sieving process (A) mean crystal size $160 \mu \mathrm{m}$, and (B) crystal size above $400 \mu \mathrm{m}$.

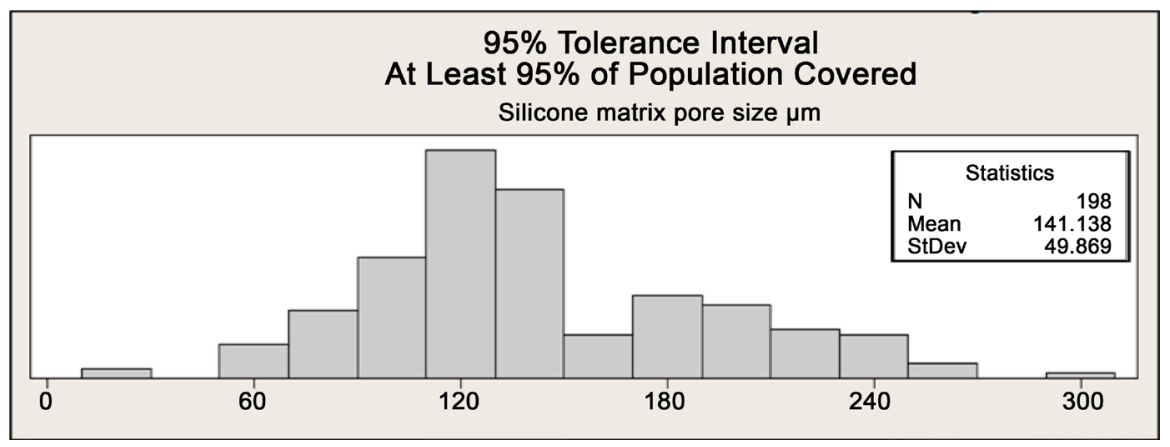

Figure 2. Pore size distribution from porous silicone matrix made with sieved sugar.
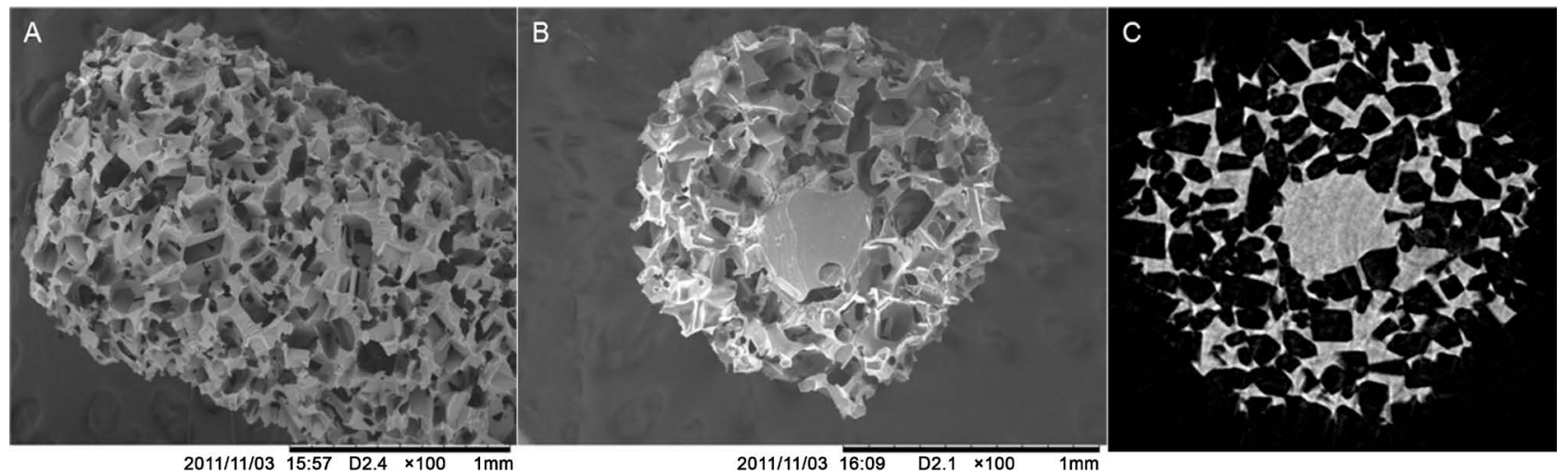

Figure 3. Micrographs from porous silicone matrix made with the sieved sugar, (A) lateral position (B) cross section and (C) binary image from micrograph $\mathrm{B}$.

microscope. Figure 3 demonstrates a good pore density and connectivity, a requirement for biotechnological and biomedical applications. The porous silicone matrix used in this research had a pore distribution size below $300 \mu \mathrm{m}$ with a mean pore size of $141 \mu \mathrm{m}$. The pore structures from the porous silicone matrix consist of irregularly shaped voids and connecting channels.

Gold and silver nanoparticles (NPs) are stabilized or protected by a shell of organic ligands and display good stability in water. Reducing agents such as car- 
bohydrate compounds, sodium ascorbate, and sodium citrate are considered reagents for synthesis in the green chemistry field. These can be extracted from natural sources using non-organic solvent chemistry [17]. Green chemistry is an excellent alternative for preparing nanostructures that have a potential use in medicine, because these chemicals are not toxic or dangerous for humans. The process for nanoparticle-porous silicone matrix formation is summarized and shown in Figure 4. Colloid metal (silver, gold) nanoparticles were synthetized with the matrices inside the reaction or metal solution. Synthetized nanoparticles were attracted and immobilized on the surface of porous silicone matrices.

Colloidal stable gold and silver nanoparticles with controllable size and remarkable stability were prepared and immobilized on silicone porous matrices. Nanoparticle stability is always an issue; many factors and their interactions are involved in metal nanoparticle size. $\mathrm{PH}$, temperature, concentration and ratio between the metal source, the stabilizer and the reducing agent all affect the particles' size.

Fluorescent X-ray spectrophotometer measurements were done to determine the thickness of gold and silver. The Y axis represents the thickness measurement in nanometers and the $\mathrm{X}$ axis represents the lot number. On all performed measurements, the gold film is above the silver film on the silicone surface.

The obtained value for silver film thickness was from 110 to $50 \mathrm{~nm}$, with an average value of 85.6 and a standard deviation of 21.8. Gold film thickness was from 25 to $75 \mathrm{~nm}$, with an average value of 36.9 and a standard deviation of 18.2, as shown in Figure 5. Each lot thickness value was the average value from ten different measurements,, ten lots, and one hundred measurements in total. This data illustrates nanoparticle deposition on the porous silicone matrix after the reaction process.

Figure 7 shows particles immobilized (bright ruby color) on the surface, characteristic after gold nanoparticle immobilization. Particles between 150 - $600 \mathrm{~nm}$ were obtained, according to the estimation made using the SEM micrographs, Figure 6.

Porous silicone matrix water uptake was measured and compared for naked samples

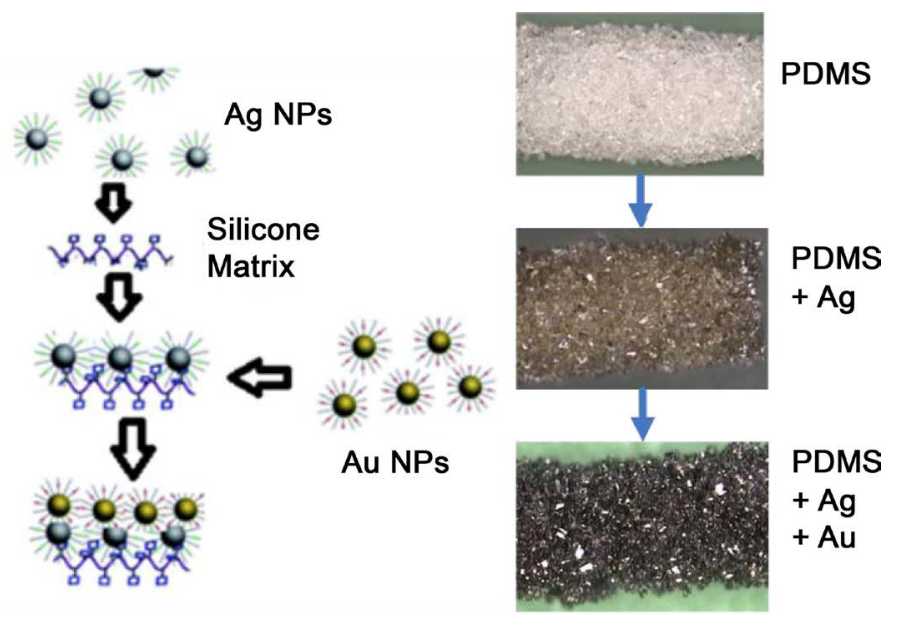

Figure 4. Gold and silver nanoparticles immobilization on porous silicone matrix surface. 


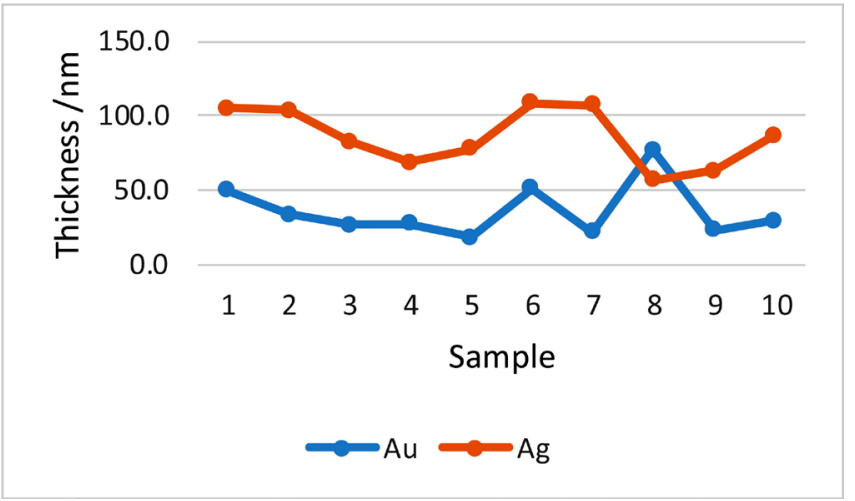

Figure 5. Gold and silver nanofilm immobilized on porous silicone matrix.

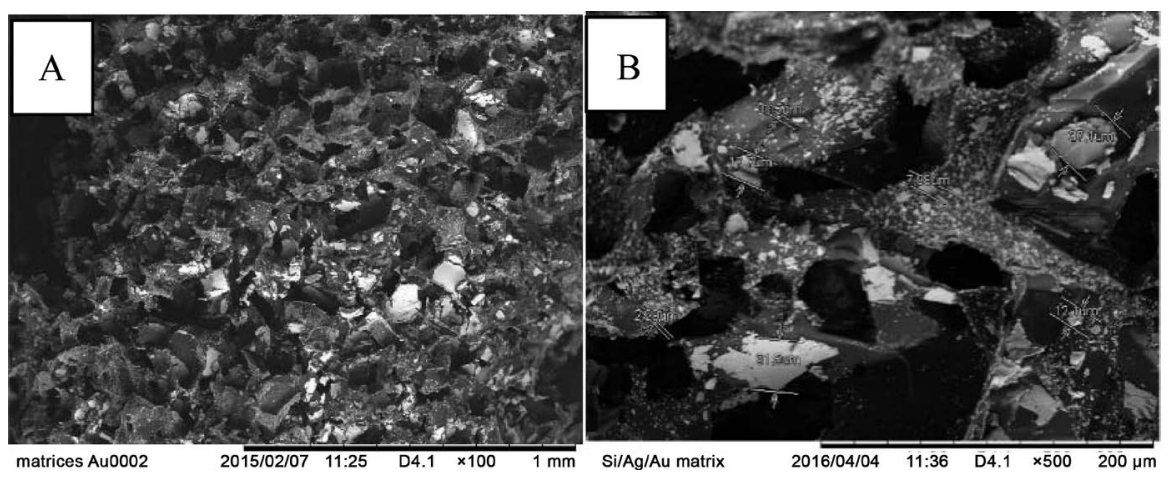

Figure 6. Micrographs from the porous silicone matrix after silver and gold nanoparticle immobilization, magnification A: $100 \times$ and B: $500 \times$.

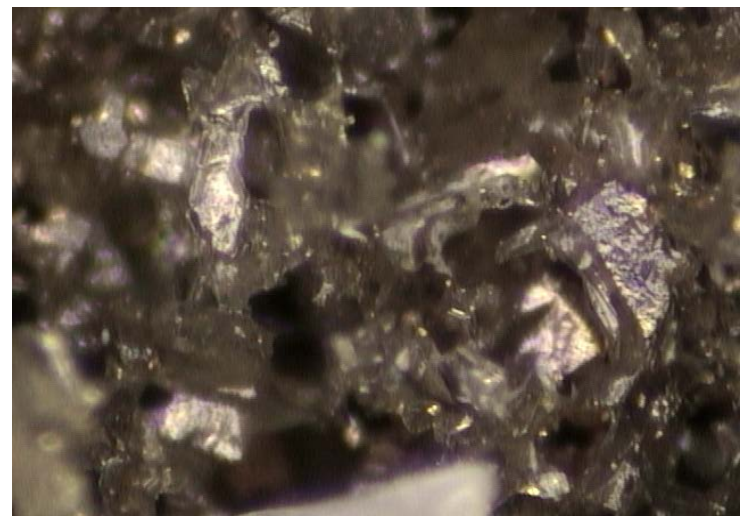

Figure 7. Optical image from porous silicone matrix after silver and gold nanoparticle immobilization, magnification $175 \times$.

and samples after immobilization of nanoparticles. Figure 8 shows the percentage of water absorbed. A higher water absorption value was obtained when the silver and gold nanoparticles were on the surface. The result obtained with respect to water absorption was gold/silver/porous silicone matrix $>$ silver/porous silicone matrix $>\mathrm{Au}$ /porous silicone matrix $>$ naked porous silicone matrix.

Water absorption depends on silicone surface energy. Silicone film without immobilized metal nanoparticles shows a higher contact angle value of $108^{\circ}$. This value agrees with the hydrophobic silicone properties (low surface energy) but the silicone 


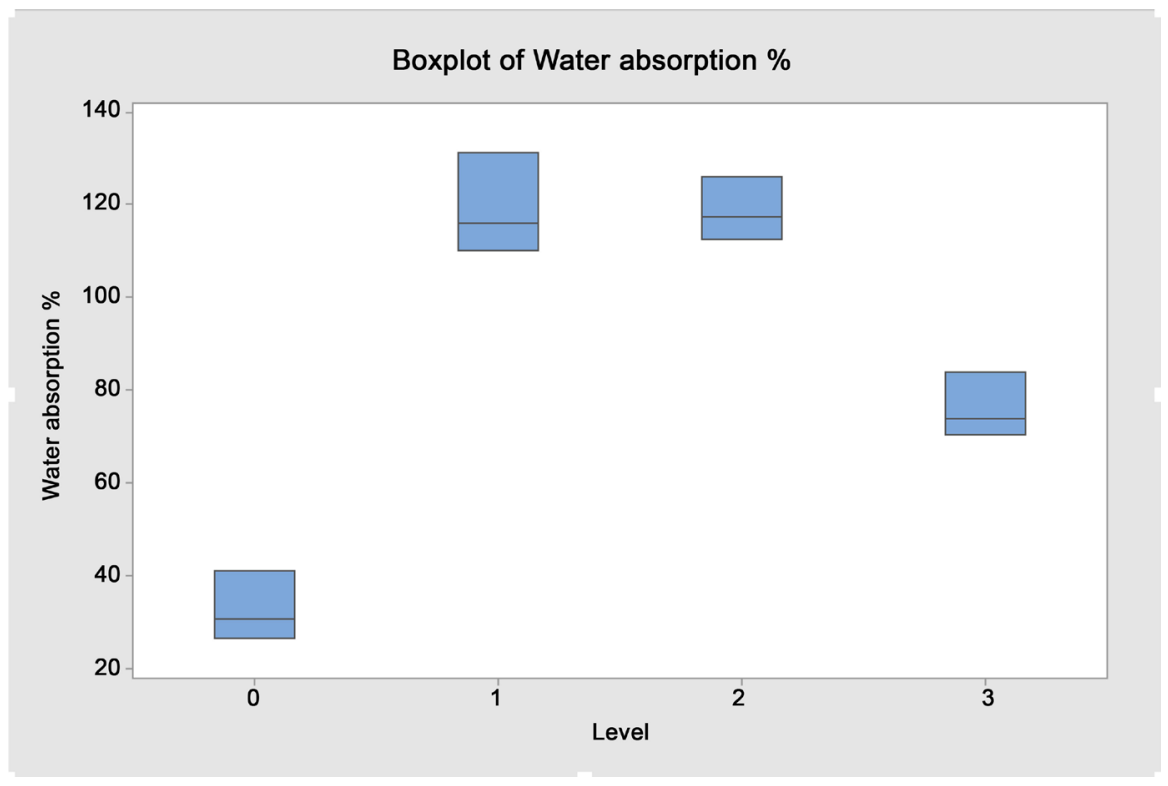

Figure 8. Water absorption percentage from porous silicone matrix after metal nanoparticle immobilization, Level 0: pure porous silicone, 1: porous silicone/Ag/Au, 2: porous silicone/Ag, and 3: porous silicone/Au.

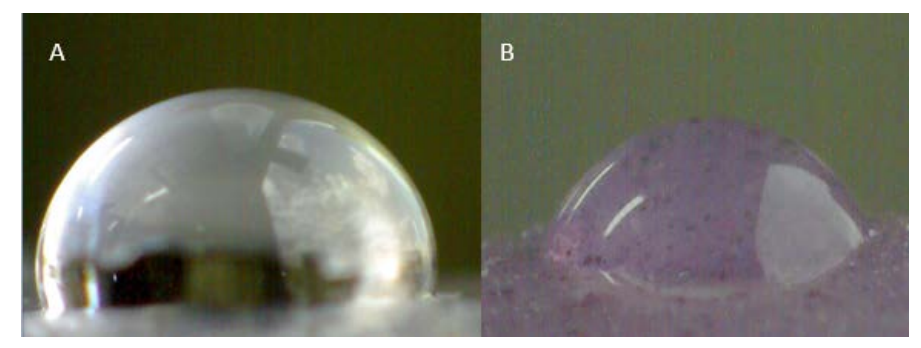

Figure 9. Water drop over silicone film: (A) Pure silicone film; (B) Silicone film with Ag/Au nanoparticles.

film with immobilized metal nanoparticles ( $\mathrm{Au}, \mathrm{Ag}$ ) shows a lower contact angle value of $70^{\circ}$, indicating higher surface energy. See Figure 9. The surface gets wet, because it turns into a hydrophilic surface. This result explains the values obtained in the water absorption test, Figure 8.

\section{Conclusion}

Sugar crystals are good template agents for producing porous silicone matrices with controlled pore size. No organic solvents or high energy processes are needed to remove a pore-forming agent. The immobilization of gold and silver nanoparticles was achieved using a colloidal suspension in contact with a porous silicone matrix. Good nanoparticle dispersion on the surface was achieved with a non-uniform nanofilm formation, as shown by SEM, optical imaging and XRF. The water absorption of a porous silicone matrix with immobilized gold and silver nanoparticles was higher than that of a naked silicone porous matrix. Therefore, porous silicone matrix water uptake is improved by $\mathrm{Ag} / \mathrm{Au}$ nanoparticle immobilization. These results are promising; the immobilization of both gold and silver nano- 
particles can improve biocompatibility and increase antimicrobial properties, through surface modification exerting a positive effect on the response of biological material.

\section{Conflict of Interest}

Authors declare that there is no conflict of interest regarding the publication of this paper.

\section{References}

[1] Jugdaohsingh, R. (2007) Silicon and Bone Health. Journal of Nutrition, Health and Aging, 11, 99-110.

[2] Sánchez-Guerrero, J., Graham, M., Colditz, A., Karlson, E., Hunter, D., Speizer, F. and Liang, M. (1995) Silicone Breast Implants and the Risk of Connective-Tissue Diseases and Symptoms. New England Journal of Medicine, 332, 1666-1670. https://doi.org/10.1056/NEJM199506223322502

[3] Moss, A., Vasilakis, C., Holley, J., Foulks, C., Pillai, K. and McDowell, D. (1990) Use of a Silicone Dual-Lumen Catheter with a Dacron. Cuff as a Long-Term Vascular Access for Hemodialysis Patients. American Journal of Kidney Diseases, 16, 211-215. https://doi.org/10.1016/S0272-6386(12)81020-1

[4] Liu, D., Shahbazi, M., Bimbo, L., Hirvonen, J. and Santos, H. (2014) Biocompatibility of Porous Silicon for Biomedical Applications, University of Helsinki, Finland, Porous Silicon for Biomedical Applications. Woodhead Publishing Limited, Cambridge, 129-181.

[5] Solano-Umaña, V. and Vega-Baudrit, J. (2015) Micro, Meso and Macro Porous Materials on Medicine. Journal of Biomaterials and Nanobiotechnology, 6, 247-256. https://doi.org/10.4236/jbnb.2015.64023

[6] Nativ-Roth, E., Rechav, K. and Porat, Z. (2016) Deposition of Gold and Silver on Porous Silicon and inside the Pores. Thin Solid Films, 603, 88-96.

https://doi.org/10.1016/j.tsf.2016.01.020

[7] Mora, M., Bornacelli, J., Nava, R., Zanella, R. and Reyes-Esqueda, J. (2014) Porous Silicon Photoluminescence Modification By-Colloidal Gold Nanoparticles: Plasmonic, Surface and Porosity Roles. Journal of Luminescence, 146, 247-255. https://doi.org/10.1016/j.jlumin.2013.09.053

[8] O’Reillya, A., Kuanb, M. and Quitorianoa, N. (2013) pH Dependent Sticking Probability of Gold Colloid on Silicon. Colloids and Surfaces A: Physicochemical and Engineering Aspects, 436, 130-132. https://doi.org/10.1016/j.colsurfa.2013.06.025

[9] Seong-Wan, R., Chang-Hoon, K., Jin-Woo, H., Chung-Jin, K., Cheulhee, J., Hyun, P. and Yang-Kyu, C. (2010) Gold Nanoparticle Embedded Silicon Nanowire Biosensor for Applications of Label-Free DNA Detection. Biosensors and Bioelectronics, 25, 2182-2185. https://doi.org/10.1016/j.bios.2010.02.010

[10] Solano-Umaña, V. and Vega-Baudrit, J. (2015) Gold and Silver Nanotechology on Medicine. Journal of Chemistry and Biochemistry, 3, 21-33.

[11] Gallo, A., Paladini, F., Romano, Al., Verri, T., Quattrini, A., Sannino, A. and Pollini, M. (2016) Efficacy of Silver Coated Surgical Sutures on bacterial Contamination, Cellular Response and Wound Healing. Materials Science and Engineering, 69, 884-893. https://doi.org/10.1016/j.msec.2016.07.074

[12] Steffensen, S., Vestergaar, M., Groenning, M., Alm, M., Franzyk, H. and Nielsen, H. 
(2015) Sustained Prevention of Biofilm Formation on a Novel Silicone Matrix Suitable for Medical Devices. European Journal of Pharmaceutics and Biopharmaceutics, 94, 305-311. https://doi.org/10.1016/j.ejpb.2015.05.014

[13] Hutcheon, G., Messiou, C., Wyre, R., Davies, M. and Downes, S. (2001) Water Absorption and Surface Properties of Novel Poly (Ethylmethacrylate) Polymer Systems for Use in Bone and Cartilage Repair. Biomaterials, 22, 667-676. https://doi.org/10.1016/S0142-9612(00)00229-5

[14] Dhandayuthapani, B., Yoshida, Y., Maekawa, T. and Kumar, D. (2011) Polymeric Scaffolds in Tissue Engineering Application. International Journal of Polymer Science, 2011, Article ID: 290602. https://doi.org/10.1155/2011/290602

[15] Solano-Umaña, V. and Vega-Baudrit, J. (2015) Porous Size Control on Silicone Matrix. International Journal of Recent Scientific Research, 6, 6290-6295.

[16] Solano-Umaña, V. and Vega-Baudrit, J. (2016) Controlled Deposition of Gold and Silver on a Porous Silicone Matrixs. Jacobs Journal of Nanomedicine and Nanotechnology, 1, 1-8.

[17] Renata, B., Sigg, L., Clift, M., Herzog, F., Minghetti, M., Johnston, B., Petri-Fink, A. and Rothen-Rutishauser, B. (2013) Bioavailability of Silver Nanoparticles and Ions from a Chemical and Biochemical Perspective. Journal of the Royal Society Interface, 10, Article ID: 20130396. 